Article

\title{
A QoS-Aware Dynamic Bandwidth Allocation Algorithm for Passive Optical Networks with Non-Zero Laser Tuning Time
}

\author{
Mohammad Zehri 1,2, Adebanjo Haastrup ${ }^{1}$, David Rincón ${ }^{1, *}$, José Ramón Piney ${ }^{1}$, Sebastià Sallent ${ }^{1}$ and Ali Bazzi ${ }^{2}$ \\ 1 Department of Network Engineering, Universitat Politècnica de Catalunya (UPC), 08860 Castelldefels, Spain; \\ mohammad.habib.zehri@upc.edu or mohamad.zehri@liu.edu.lb (M.Z.); adebanjo.haastrup@upc.edu (A.H.); \\ david.rincon@upc.edu (D.R.); jose.ramon.piney@upc.edu (J.R.P.); sallent@entel.upc.edu (S.S.) \\ 2 Department of Computer and Communication Engineering, Lebanese International University (LIU), \\ Beirut 14404, Lebanon; ali.bazzi@liu.edu.lb \\ * Correspondence: david.rincon@upc.edu; Tel.: +34-93-413-7056
}

Citation: Zehri, M.; Haastrup, A.; Rincón, D.; Piney, J.R.; Sallent, S.; Bazzi, A. A QoS-Aware Dynamic Bandwidth Allocation Algorithm for Passive Optical Networks with Non-Zero Laser Tuning Time. Photonics 2021, 8, 159. https://doi.org/10.3390/ photonics 8050159

Received: 30 March 2021

Accepted: 30 April 2021

Published: 10 May 2021

Publisher's Note: MDPI stays neutral with regard to jurisdictional claims in published maps and institutional affiliations.

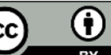

Copyright: $\odot 2021$ by the authors. Licensee MDPI, Basel, Switzerland. This article is an open access article distributed under the terms and conditions of the Creative Commons Attribution (CC BY) license (http://creativecommons.org/licenses/by/4.0/).

\begin{abstract}
The deployment of new 5G services and future demands for 6G make it necessary to increase the performance of access networks. This challenge has prompted the development of new standardization proposals for Passive Optical access Networks (PONs) that offer greater bandwidth, greater reach and a higher rate of aggregation of users per fiber, being Time- and Wavelength-Division Multiplexing (TWDM) a promising technological solution for increasing the capacity by up to $40 \mathrm{Gbps}$ by using several wavelengths. This solution introduces tunable transceivers into the Optical Network Units (ONUs) for switching from one wavelength to the other, thus addressing the ever-increasing bandwidth demands in residential broadband and mobile fronthaul networks based on Fiber to the Home (FTTH) technology. This adds complexity and sources of inefficiency, such as the laser tuning time (LTT) delay, which is often ignored when evaluating the performance of Dynamic Bandwidth Allocation (DBA) mechanisms. We present a novel DBA algorithm that dynamically handles the allocation of bandwidth and switches the ONUs' lasers from one wavelength to the other while taking LTT into consideration. To optimize the packet delay, we introduce a scheduling mechanism that follows the Longest Processing Time first (LPT) scheduling discipline, which is implemented over the Interleaved Polling with Adaptive Cycle Time (IPACT) DBA. We also provide quality of service (QoS) differentiation by introducing the Max-Min Weighted Fair Share Queuing principle (WFQ) into the algorithm. The performance of our algorithm is evaluated through simulations against the original IPACT algorithm, which we have extended to support multi-wavelengths. With the introduction of LPT, we obtain an improved performance of up to $73 \%$ reduction in queue delay over IPACT while achieving QoS differentiation with WFQ.
\end{abstract}

Keywords: passive optical networks; laser tuning time; TWDM; dynamic bandwidth allocation; optical access networks; scheduling

\section{Introduction}

Data traffic is increasing massively as the type of services is changing interactively towards media and streaming. It has been predicted that connected devices will grow significantly to reach 100 billion devices by year 2025 [1]. Passive Optical Networks (PON) are one of the major driving forces behind residential broadband access and 5G networks [2] (for example, in cloud radio access networks (C-RAN) [3,4]), as they meet the everincreasing demand for bandwidth-intensive applications such as ultra-high-definition $\mathrm{TV}$, immersive video, and the stringent end-to-end latency required by mission-critical applications.

PON is a cost-effective optical technology that provides the advantage of using passive network elements to connect users in access networks. They consist of a central unit 
called an Optical Line Terminal (OLT) at the central office of the internet service provider, and it connects through optical fiber to several Optical Network Units (ONUs) located in-or close to-the customers' premises within a $20 \mathrm{~km}$ range [5]. The optical fiber cable is shared by introducing passive optical splitters into the optical distribution network (ODN) located between the OLT and the ONUs, by which it reaches up to 64 users [6] (although some architectures allow split ratios of up to 1024 [7]). In the upstream direction, the splitter combines the upstream wavelengths from the ONUs to the OLT [8]. The PON architecture is referred to as a point-to-multipoint (P2MP) system [9]. It is a very costeffective and easy-to-manage solution, as it does not require any active electronic devices between the OLT and the ONUs [10].

The PON system is based on a shared model that allows bi-directional communication between the ONUs and the OLT. The downstream traffic is broadcast from the OLT to all ONUs while the upstream communication from the ONUs to the OLT is achieved using a time-sharing principle [8]. Owing to the shared nature of the PON, and ONU systems competing for network capacity, a mechanism must be put in place to control the allocation of the upstream transmission capacity in real time, thus avoiding data collision if two or more ONUs transmit simultaneously towards the OLT. PONs employ a dynamic bandwidth allocation (DBA) algorithm to orchestrate the allocation of network resources in the shared medium. One of the main requirements of a DBA is that it satisfies the low latency and huge bandwidth requirements of emerging applications [11].

The new generation of PON technology is based on the Time- and Wavelength-Division Multiplexing (TWDM) technique, which has been described as an evolutionary step that allows using multiple wavelengths to increase the capacity of the PONs [12]. TWDM is a hybrid technique that combines Wavelength-division multiplexing (WDM) capacity expansion with the inherent resource granularity of a Time-division multiplexing (TDMPON) to meet the growing demands for bandwidth, reach and aggregation [13]. Some TWDM-PON proposals, based on four wavelengths typically have a maximum throughput capacity of $40 \mathrm{Gbps}$, thus meeting the requirements of the NG-PON2 standards $[4,14]$. TWDM-PON is used as a major application in mobile fronthaul networks for connecting the centralized baseband unit (BBU) and remote radio heads (RRHs) in 5G C-RANs, which have extreme requirements in terms of capacity, latency, and cost-efficiency [15,16].

In TWDM-based PONs, the resource allocation process in the upstream link is twodimensional, consisting of wavelength and time slot allocation. The DBA scheme dynamically allocates the wavelengths (typically four) among the ONUs and shares available bandwidth in terms of time slots among the ONUs in the upstream link. An important characteristic of TWDM-PONs is the use of tunable transceivers at the ONUs [5], which are thus enabled to switch their wavelengths. It is important for the DBA to efficiently handle the assignment of the wavelengths, which involves the switching of ONUs from one wavelength to the other. The wavelength assignment decision is communicated to the ONUs by the OLT, and the ONUs can transmit their frames at their allotted time slots on the assigned wavelength [5]. This approach makes it necessary for ONUs to change their wavelengths to optimize the use of the shared medium. ONUs use tunable lasers to facilitate the switching of the wavelengths as instructed by the OLT, thus adding both complexity and a Laser Tuning Time (LTT) delay that may have a great impact on the performance of the system [4,5]. Only a few research works consider LTT when designing or evaluating the performance of DBAs for TWDM networks $[17,18]$. It is therefore, necessary to develop more sophisticated DBA algorithms that will ensure fair distribution of resources among ONUs while taking into consideration the delays caused by lasers switching between wavelengths.

In this paper, we propose a novel DBA algorithm to efficiently manage the allocation of bandwidth and wavelength assignment while considering the LTT delay. Transmitting on multi-wavelength PON poses the problem of scheduling with a constraint on the completion time and an overall effect on the delay. Therefore, we aim to reduce the queue delay by introducing a scheduling scheme based on the Longest Processing Time first 
(LPT) principle [19]. The goal of LPT is to minimize the maximum completion time for processing and transmitting the requests from the ONUs. This is achieved by the OLT sorting the ONUs' bandwidth requests in descending order, with the largest request being processed first. Finally, we introduce weight-based QoS differentiation following the Max-Min Weighted Fair Share principle [20] to ensure a guaranteed bandwidth for demands requested by the users since traditional IPACT algorithm does not guarantee QoS [21].

The main contribution of our TWDM-DBA is to effectively reduce end-to-end delay, and efficiently utilize the bandwidth while achieving QoS differentiation. We validated our algorithm by comparing it with the traditional IPACT algorithm, which has been extended to use up to four wavelengths. The performance metrics of our study include queue delay and throughput. The results show that our proposed DBA can significantly improve network performance in terms of queue delay and throughput while adding QoS differentiation.

The remainder of the paper is organized as follows. Related work and the state of the art are summarized in Section 2. Section 3 introduces the proposed TWDM-DBA algorithm. Section 4 describes a performance evaluation of the proposed approach using simulation results. Conclusions and future work are described in the last section.

\section{Related Work}

\subsection{PON Standards}

The International Telecommunication Union (ITU) and the Institute of Electrical \& Electronics Engineers (IEEE) are active players that have been at the forefront of developing PON standards over the past twenty years [22].

IEEE introduces the concept of Ethernet over the shared media of Passive Optical Networks, called Ethernet PON (EPON) standards [8]. These standards fall within the IEEE 802.3 standards series [23], and they have continued to evolve. The initial gigabit speeds of EPON provides $1 \mathrm{~Gb} / \mathrm{s}$ offering a symmetric service to 64 customers with one strand of fiber from the central office (CO) to end users over a maximum distance of 20 $\mathrm{km}$ [24]. Later, 10G-EPON standardized the symmetric service at $10 \mathrm{~Gb} / \mathrm{s}$ [25]. One of the latest versions of the standards is known as NG-EPON under the IEEE 802.3ca standards and aims to support more capacity, $25 \mathrm{~Gb} / \mathrm{s}$ and $50 \mathrm{~Gb} / \mathrm{s}$ on $25 \mathrm{~Gb} / \mathrm{s}$ serial streams and improve data transmission efficiency in the access network by using multiple wavelengths [26].

The ITU developed its variant of PON known as Gigabit-capable Passive Optical Networks (GPON), which handles Asynchronous Transfer Mode (ATM) packets and GEM (GPON Encapsulation Method) frames while providing QoS assurance and 2.5/1.25 Gb/s asymmetric service [22]. The latest version of ITU GPON standards is ITU-T G.989.2, which specifies Next-Generation Passive Optical Network 2 (NG-PON2) [27]. It has three types of channel rates in each of the wavelengths: 10/2.5 Gbps, 10/10 Gbps, and 2.5/2.5 Gbps-downstream and upstream. NG-PON2 introduces time and wavelength division multiplexing (TWDM), which aggregates multiple wavelengths to achieve increased capacity with a nominal aggregate capacity of $40 \mathrm{Gbps}$ in the downstream direction and 10 Gbps in the upstream direction $[27,28]$. The G.HSP project under the ITU-T Study Group 15 is currently working on providing $50 \mathrm{~Gb} / \mathrm{s}$ on a serial stream.

For a long time the efforts of the standardization groups have focused on increasing the rate (from 1 to $50 \mathrm{Gbps})$, maintaining the same aggregation rate (16/32/64 customers) and the same reach between the central office (CO) and the customers. To avoid the reach limitation, the ITU-T proposes to use mid-span reach extenders that involve deploying remote active cabinets resulting in an additional deployment and maintenance cost. This solution has been standardized for G-PON [29] and XG(S)-PON [30]. The limitation of the aggregation rate is not limited by the upper layers of protocols, which in XG(S)-PON can 
manage up to 1024 ONU identifiers, but by the limitations of the physical layer, and specifically the power budget.

To overcome the current reach and aggregation limitations of PON networks, a working group was formed in November 2018 under the auspices of IEEE, the P802.3cs "SuperPON" [25]. Subsequently, the ITU-T initiated the ITU-T Q2/SG15 working group and approved the standardization of Super-PON in 2019 that will be defined in ITU-T recommendation G.9807.3 [31]. Under the Super-PON umbrella, it is proposed to create a PON network with a range of up to $50 \mathrm{~km}$, with a coverage of 1024 customers per fiber over a passive optical distribution network (ODN). In the optical layer, the Super-PON will carry multiple optical carriers over one fiber strand which will allow multiple PON instances to be mapped to different carriers. This is possible by combining WDM and TDMA technology, optical amplification and multiplexing performed in the Central Office (CO), wavelength routing in the ODN and the fully tunable transmitters in the ONU [32].

\subsection{Laser Tuning Time}

Tunable optical components in the ONUs enable extensive wavelength flexibility and allow the ONUs to change from heavily loaded wavelengths to idle ones in order to reduce delay and create resource allocation balance in the system [11]. There are several types of tunable lasers, which are categorized based on the speed at which they can switch wavelengths [4,5]. Three classes are defined by ITU-T [27], as shown in Table 1. The lasers can switch between wavelengths within a few microseconds and one second. While the slow lasers are considerably cheaper, the fast lasers are very costly and energy-consuming [12]. Some solutions for a fully tunable ONU transmitter are distributed Bragg reflector (DBR) lasers and Distributed feedback (DFB) lasers. This second solution is more developed and has a lower cost, although due to problems in temperature stabilization, in WDM systems, it is common to limit tuning to four channels [32].

Table 1. Classes of Laser Tuning Time, according to ITU-T [23].

\begin{tabular}{cc}
\hline Class & Laser Tuning Time \\
\hline Class 1 & $<10 \mu \mathrm{s}$ \\
Class 2 & $10 \mu \mathrm{s}$ to $25 \mathrm{~ms}$ \\
Class 3 & $25 \mathrm{~ms}$ to $1 \mathrm{~s}$ \\
\hline
\end{tabular}

\subsection{DBAs for TWDM-PONs}

The issue of the OLT allocating bandwidth to the ONUs is resolved by following a layered approach for job scheduling, as proposed in [33]. The scheduling concept can be divided into scheduling framework and scheduling policy. The scheduling framework deals with the OLT making scheduling decisions for job processing, and the scheduling policy addresses how the DBA allocates time and wavelengths to the ONUs. There are three ways of implementing the scheduling framework: Online, Offline, and Just-in-Time. Online scheduling allows bandwidth to be allocated to ONUs' jobs as soon as they are received at the OLT. Since requests are granted to the ONUs immediately without waiting to consider requests from other ONUs, the system is considered unfair. In offline scheduling, decisions are made after all the requests from the ONUs are received at the OLT, thereby assuring fairness in the system. The downside of the offline scheme is the delay and the underutilization of the link during the time when the OLT receives the report from the first ONU and the time when it issues the grant. To address the aforementioned problems, Just-in-Time scheduling was proposed by [33] to allow the OLT to postpone decision-making until one channel is about to become idle. The decision-making in Just-in-Time scheduling occurs later than in online scheduling and sooner than in offline scheduling.

Many studies have been carried out on the dynamic bandwidth allocation (DBA) algorithms for TWDM-PONs. The DBA algorithms in TWDM-PON are two-dimensional, 
dynamically combining bandwidth and wavelength allocation for the ONUs in the PON system. This DBA scheme is referred to as Dynamic Wavelength and Bandwidth DWBA algorithms [17]. DWBA allows dynamic wavelength and dynamic time slots allocation to the ONUs by maintaining information about each wavelength channel and determines the transmission period assigned to each ONU on a specified wavelength channel [8]. DWBA can be implemented by using either separate time and wavelength scheduling (STWS) algorithms or joint time and wavelength scheduling (JTWS) algorithms [12]. STWS algorithms decouple the wavelength assignment from the time slot allocation and are thus simple. JTWS algorithms, on the other hand, combine the wavelength assignment with time slot allocation. JTWS is more efficient and scalable but more complex than STWS $[34,35]$. The work of [36] decouples the wavelength assignment from the Time-Division Multiple Access (TDMA) assignment and removes scheduling complexity by using an adaptive threshold, thus leading to a lower processing requirement. An adaptive wavelength allocation pattern for scheduling multi-wavelength ONUs in NG-EPON is proposed in [37] to achieve a low packet-loss ratio. The wavelength allocation pattern is based on an adaptive threshold that reflects both the ONU's absolute bandwidth request size and the relative bandwidth request size sent to other ONUs.

Some of the recent works on DBA concern specific network architectures. The work of [4] is on mobile fronthaul, with attention given to the bursty nature of its traffic, and proposes a DBA able to satisfy the strict latency requirements. A Dynamic Wavelength and Bandwidth Allocation (DWBA) scheme for TWDM-PON is proposed in [11] to satisfy the strict delay requirement for fronthaul with a minimum number of active wavelength channels. It minimizes active wavelength channels by considering the high burstiness of fronthaul data transmission and using the difference in the propagation delay between the OLT and ONUs. A low latency DBA scheme for NG-PON2 to support both 5G fronthaul services and data services is proposed in [38]. The DBA splits the upstream frame into sub-frames of equal duration, and each ONU is allocated with a time slot in each subframe without specifying an allocation interval for the associated queue.

The DBA in TWDM-PON requires wavelength tuning functions; however, most of the dynamic wavelength assignment algorithms do not consider the tuning time. Wavelength tuning reduces channel utilization and increases packet delay [18]. The work of [17] is, to the best of our knowledge, the first to consider the laser tuning time in the design of the DBA. It notes that proper DBA algorithms should maximally exploit the statistical gain among requests, under the condition that lasers are given enough time to switch wavelengths. A DBA based on a minimum wavelength tuning (MWT) scheme is proposed in [39]. The scheme minimizes the frequency of wavelength tuning to reduce packet delay and improve channel utilization. The DBWA proposed in [18] manages transceivers with tunable lasers that have different LTT values combined with transceivers that have nontunable (fixed) lasers in a single transmission. A multi-tuning-time ONU scheduling (MOS) algorithm that uses an adaptive scheduling algorithm for the coexistence of ONUs with different tuning time in virtual PON is proposed in [40]. The MOS algorithm is able to reduce waste of bandwidth resources and achieve load balancing.

Concerning the quality of service (QoS) in TWDM-PON, some works have recently started to appear in the literature, but they are very limited. A high-priority first dynamic wavelength and bandwidth allocation algorithm in TWDM-PON is proposed in [41]. The DWBA is implemented using five types of transmission containers (T-CONTs), and it can execute four kinds of bandwidth strategies to effectively reduce the average delay, slightly improve bandwidth utilization, and ensure greater fairness for the diverse types of traffic. A QoS-based DWBA for multi-scheduling domain EPON (MSD-EPON) is proposed in [8] to arbitrate the channel bandwidth efficiently in NG-EPON. Following an innovative approach according to the modern traffic requirements, it uses an adequate technique by deploying a blend of online-offline according to the traffic types. A max-min fair allocation scheme is introduced into the algorithm proposed in [42] to provide a minimum level 
of service in every frame. This technique uses a combination of status reporting and traffic monitoring techniques to achieve fairness and a significant decrease in the average delay.

Existing DBA algorithms as discussed above, address different individual and specific problems such as delay, bandwidth utilization and QoS etc. in PONs without any of them addressing all the issues collectively. There is therefore, a need for a new DBA algorithm to be developed taking LTT and QoS into consideration while minimizing the delay and efficiently utilizing the bandwidth. Our work is based on IEEE EPON standards while drawing on several ideas, such as multiwavelength capability and the associated LTT delay introduced in ITU standards under NG-PON2, which allows ONUs to change their wavelengths. Our contributions align with the work of $[17,39]$ on the application of LTT in DBA algorithms, and we go further by focusing on maintaining a balance between the switching of the ONUs' wavelengths and the associated delay, thus allowing us to achieve optimal bandwidth utilization. We reduce the frame makespan and minimize the delay by introducing the LPT scheme, which is a member of the bin-packing method that is similar to the MULTIFIT used on IPACT in [17] for scheduling the requests from the ONUs.

Additionally, we apply a weight-based bandwidth guarantee scheme in accordance with the Max-Min Weighted Fair Share principle in order to assure QoS differentiation in multi-wavelength PONs like NG-PON2. The Max-Min Weighted Fair Share principle is based on maximizing the minimum share of the ONU whose demand has not been satisfied. Our proposal extends the basic max-min fair allocation proposed in [42] by using priority based on weight in order to capture users' service requirements and weights accordingly. To the best of our knowledge, this is the first time an LTT-aware DBA is enhanced with LPT and WFQ.

\section{The Proposed Algorithm}

Our proposal builds on IPACT DBA [21], an online algorithm that follows an interleaved polling scheme to schedule transmission from the ONUs in a centralized approach. The requests from the ONUs are sent to the OLT, which has complete knowledge of the queue of the ONUs and when the last bit will arrive. With this knowledge, the OLT will start scheduling the grant for the next ONU. Since the OLT does not have to wait for the rest of the ONUs' requests to reach the OLT before it starts processing them, the waiting time is reduced and the overall delay is minimized.

The original IPACT algorithm has been extended with the capability of coping with multiple wavelengths of the TWDM-PON in [43]. Optimally scheduling the requests from the ONUs on the four wavelengths in TWDM-PON is a problem similar to the scheduling of computational tasks in a multiprocessor environment with identical processors acting in parallel. Mapping this environment with multiprocessor scheduling, with wavelength channels as machines and ONUs' requests as jobs, is indicative of an NP-hard optimization problem, which is computationally prohibitive [44]. Given a set J of jobs where job $J_{i}$ has length $L_{\mathrm{i}}$ and several wavelengths $\omega$, our objective is to achieve the earliest possible time required to schedule all jobs in $J$ on $\omega$ wavelengths such that none overlaps. Since there is a large number of requests coming from the ONUs to be transmitted on the four wavelengths in real-time, heuristic approaches are most suitable in achieving near-optimal scheduling efficiency [19].

We introduce the LPT scheduling algorithm, due to its simplicity, to solve the problem of scheduling the requests on multiple wavelengths to achieve minimal makespan of the requests' processing [45,46]. LPT is a non-preemptive scheduling algorithm that uses the priority to schedule requests to achieve near-optimal efficiency. LPT allows the sorting of the requests made to the OLT during a cycle $i$ by the ONUs $J_{1}(i), J_{2}(i) \ldots J_{M}(i)$, according to the length of time needed for them to be processed such that $L_{r}(i) \geq L_{s}(i) \geq \ldots \geq L_{m}(i)$ being $\mathrm{r}, \mathrm{s}$, and $m \leq M$. LPT has the advantage of scheduling almost equal loads on the wavelengths and avoiding situations where some wavelengths will be idle. The upper limit of 
LPT, $\frac{C_{\max }(L P T)}{C_{\max }(O P T)^{\prime}}$, has the approximation ratio shown in (1) where $C_{\max }(L P T)$ is the maximum makespan of LPT heuristic, and $C_{\max }(O P T)$ is the maximum makespan of an optimal scheduler [47].

$$
\frac{C_{\max }(L P T)}{C_{\max }(O P T)} \leq \frac{4}{3}-\frac{1}{3 M}
$$

At the beginning of each cycle, the algorithm acknowledges the number of connected ONUs whose queues are not empty. Based on the lengths of the jobs, the jobs reported from connected ONUs $\left(J_{m}\right)$ are sorted in descending order. The ONUs are then assigned to the respective available wavelengths $\omega$ such that ONU $m$ with job $J_{m}(i)$ with the longest processing time $L_{m}(i)$ is processed first and followed by the next one, assigned to the minimally loaded channel. If $\sum_{0}^{M} L_{m} \leq \delta_{\max }$, the requested time is granted for the connected ONUs in a cycle $(i)$, else $\delta_{\max }$ will be granted and certain jobs with lower lengths have to wait for the cycle $(i+1)$. The aforementioned parameters are summarized in Table 2, and the pseudocode is provided in Algorithm 1.

Table 2. Parameters of the LTT-aware QoS based algorithm.

\begin{tabular}{cc}
\hline Parameter & Description \\
\hline$M$ & Total number of ONUs \\
$\Omega$ & Assigned wavelength, $0 \leq \omega \leq 3$ \\
$i$ & Cycle number $\mathrm{i}, 0<i<\infty$ \\
$J_{m}(i)$ & The job requested by ONU m at cycle $i, 1<m<M$ \\
$L_{m}(i)$ & Length of job $J_{m}(i)$ requested by ONU m at cycle $i$ \\
$T$ & Laser tuning time \\
$\omega_{i}$ & The wavelength assigned for ONU $m$ during cycle $i$ \\
$\theta(\omega, i)$ & Waiting time for a job $J_{m}(i)$ on a wavelength $\omega$ during cycle $i$ \\
$\delta_{m a x}$ & Maximum allowed cycle length in bytes \\
$\varphi_{\omega}$ & The completion time of the last job on wavelength $\omega$ \\
$\alpha_{m}$ & Weight of the ONU $m$ \\
$\alpha_{\min }$ & The smallest weight among ONUs \\
$\Gamma$ & The summation of the normalized weights \\
$P$ & Weighted base resource share for an ONU \\
$k$ & Number of connected ONUs in cycle $i$ \\
$\beta_{m}$ & Resource share based on the weight of each user \\
\hline
\end{tabular}

Furthermore, to guarantee fairness in the sharing of resources as IPACT has no inherent QoS mechanism, we introduce QoS guarantees based on Weighted Fair Queuing (WFQ) scheme in accordance with the Max-Min Weighted Fair Share principle [48] for weight-based differentiation of users. WFQ is a discrete implementation of the generalized processor sharing (GPS) policy and an extension of fair queueing. It is realistically assumed that users have different bandwidth needs with varying priorities, therefore, all the ONUs do not request for an equal share of the resources at every given cycle. Consequently, allocating equal resources to them will lead to a waste of resources by the ONUs whose demands are lower than allocated grants, and some ONUs with higher requests will not be satisfied. Accordingly, some ONUs that have higher bandwidth demands are given more weight compared to ONUs with lower bandwidth demands and they are thus allocated relatively higher resources.

As shown in the pseudocode provided in Algorithm 2, we associate weights $\alpha_{1}, \alpha_{2}$, $\ldots, \alpha_{m}$ with ONUs 1, 2, .., $m$, which reflect their relative resource share. The resources are allocated to the ONUs in increasing order of their requests, normalized by their weights, with the small requests being fully granted first. In this case, the ONU with the lowest demand is maximized, if satisfied, only then the ONU with the second-lowest demand 
will be maximized. After the ONU with the second-lowest is satisfied, only then the ONU with the third-lowest demand will be maximized, and so on. Therefore, no ONU gets more than its demand, and the ONUs whose demands are not met get a fair share of the resources in proportion to their weights. This also avoids the situation where the resources will be monopolized by ONUs with bigger requests and consequently eliminating network congestion to some extent. We combine the WFQ principle with the LPT algorithm to give us WFQLPT, a hybrid algorithm that provides inherent QoS with the minimal makespan associated with LPT.

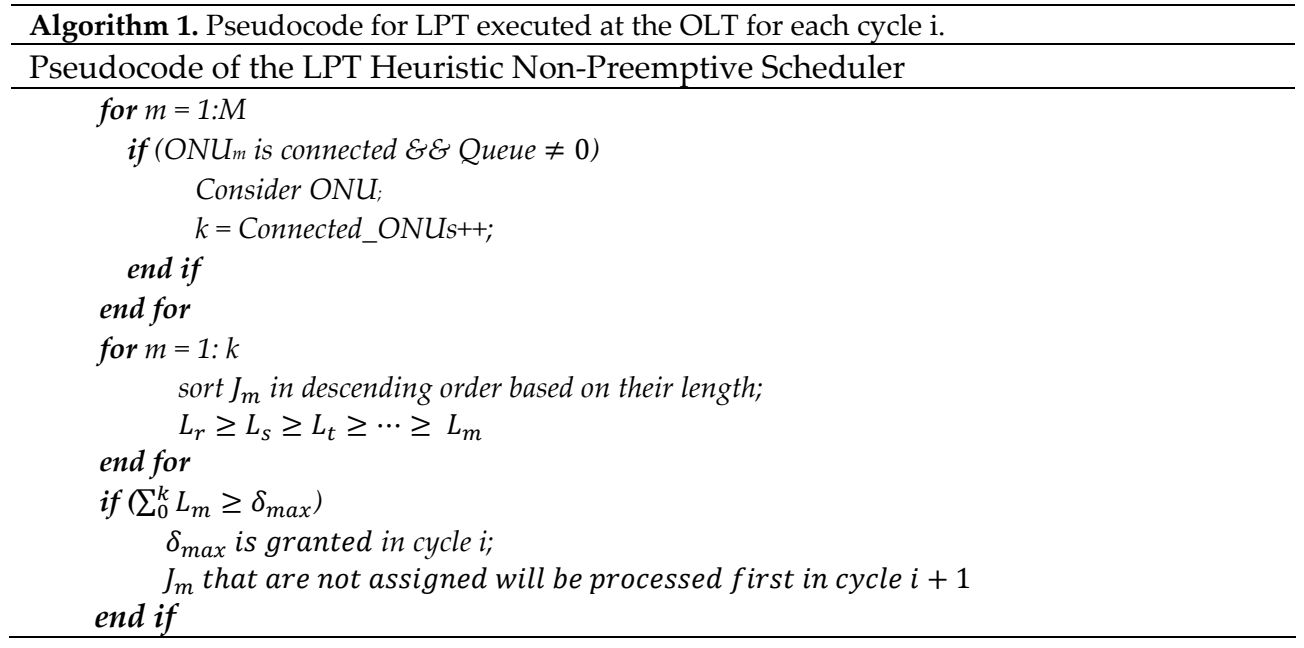

Regarding the assignment of wavelength $(\omega)$, our proposed DBA algorithm combines the time allocation and wavelength algorithms following the JTWS scheme previously described in [12]. Once the OLT receives the requests from all connected ONUs by following an offline scheduling framework, it sorts the jobs according to the LPT scheme and thereafter assigns wavelengths in accordance with the Next Available Supported Channel (NASC) scheduling policy [33]. This allows the ONUs to be assigned to the next available wavelength, where their requests will be granted. The choice of NASC aligns with the principles of the LPT scheme, in which the unassigned task with the largest computation time is assigned to the next available wavelength [49].

The assignment of the wavelength according to NASC occurs in offline scheduling mode. The offline scheduling framework gives room for the LPT scheme and allows for applying WFQ QoS differentiation as scheduling decisions are made with full knowledge of all the jobs to be scheduled for a particular scheduling cycle. The cycle is the time difference between two consecutive allocation decisions. A profound advantage of the offline scheduling framework is the increased level of scheduling control, by which the OLT differentiates QoS. Specifically, the OLT adds all the ONUs with REPORT messages into a scheduling pool, and the scheduling is done after the OLT has sorted the REPORT messages and prioritized the ONUs based on their respective QoS. The channel is considered busy until the end of the last scheduled reservation, and then the procedure is applied for considering LTT when deciding whether or not to tune the supported wavelengths. Therefore, when a wavelength becomes free, it is assigned to the ONU with the longest job in the pool, as shown in Algorithm 3. 


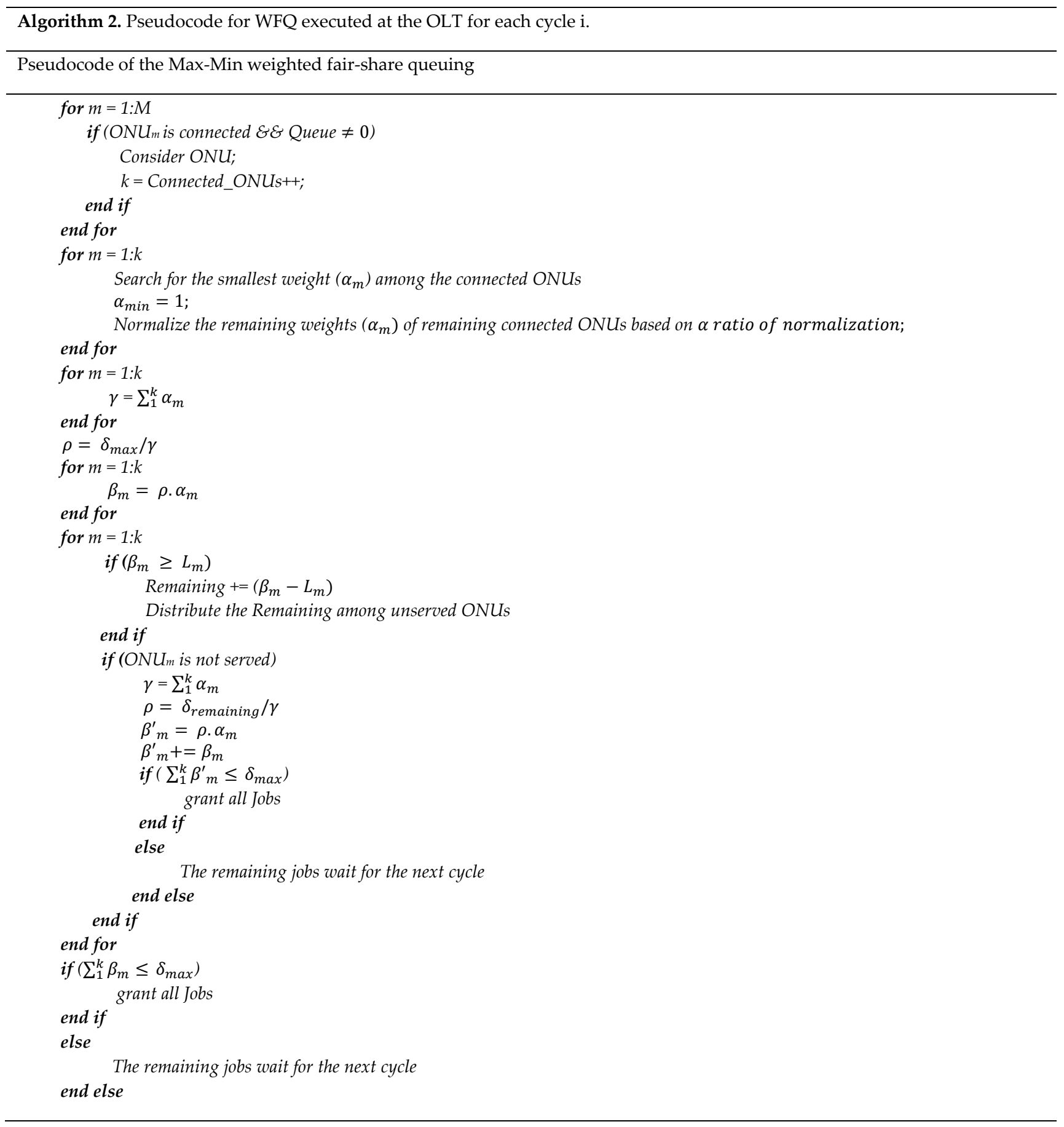




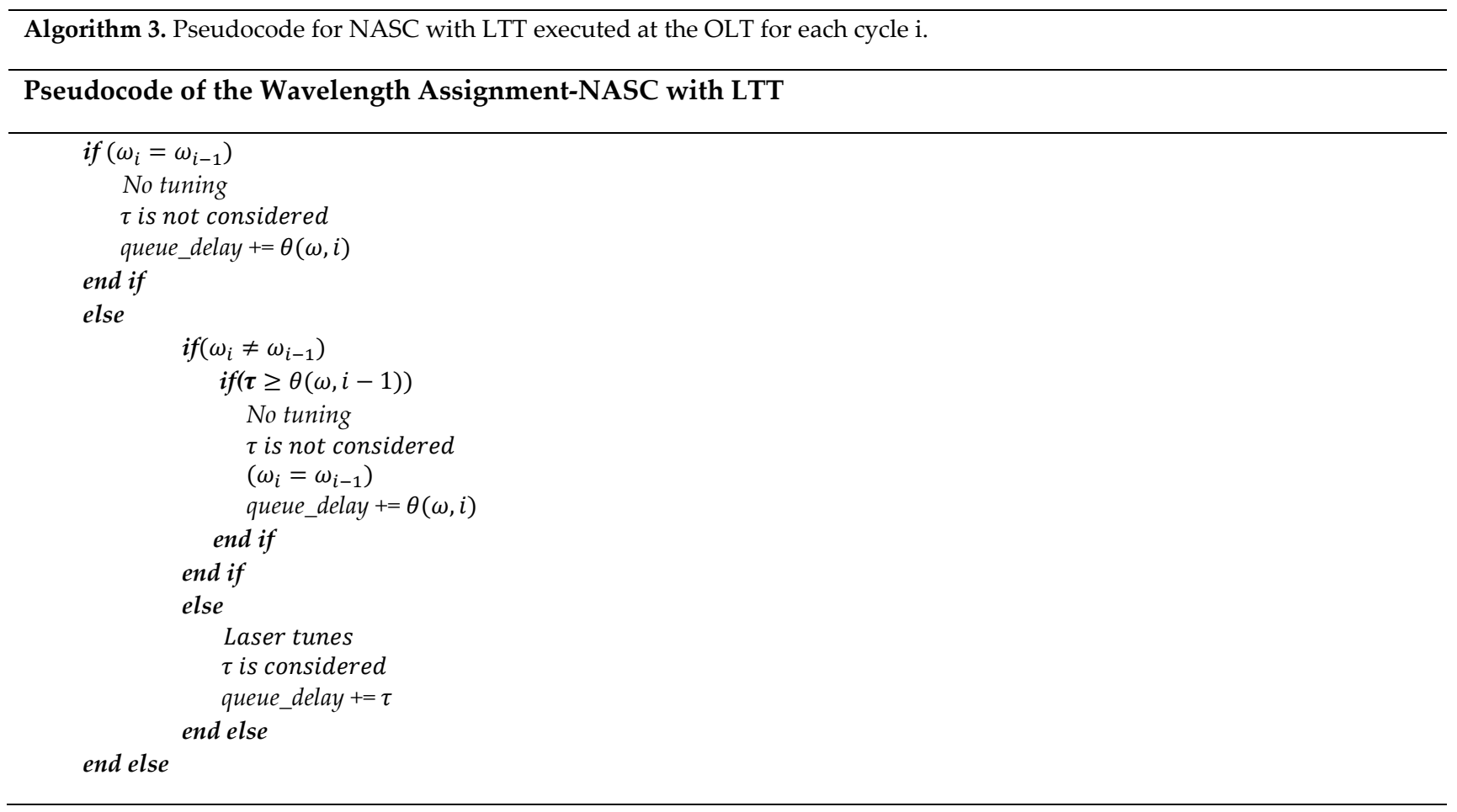

Our algorithm sorts the requests from the ONUs at the OLT according to the length of time needed for them to be processed, in descending order according to the LPT principle. The OLT sends grant messages (GATE) to the ONUs and schedules the ONU with the longest processing time first, which is then transmitted on the next available wavelength. We introduce the concept of LTT, and if the wavelength that the ONU is currently tuned to is the same as one that has been newly assigned by the OLT, then no laser tuning time is added. As shown in Algorithm 3, if the newly assigned wavelength is different from the current wavelength, the ONU checks the time needed for its current wavelength to become free and adds the laser tuning time to it. If the time needed to tune to a new wavelength is more, the ONU will remain on its current wavelength and no tuning time delay will be added. If the time to tune to a new wavelength is less, the ONU will tune to the newly assigned wavelength, and the tuning time delay will be added. This process happens continuously whenever GATE and REPORT messages are exchanged during the lifecycle of the communication between the OLT and the ONUs. Figure 1 illustrates the steps in the application of our algorithm.

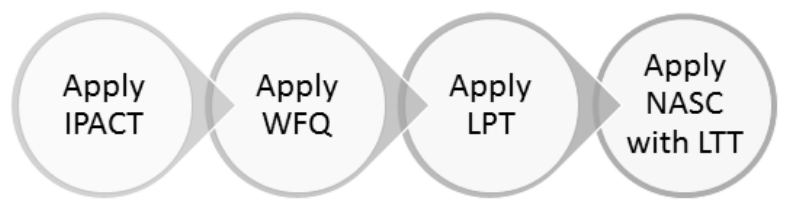

Figure 1. WFQLPT DBA algorithm sequence diagram.

\section{Performance Evaluation}

In this section, we evaluate the performance of our DBA algorithm. To validate the efficiency of our algorithm, we carried out extensive simulations using OPNET Modeller under different conditions. 


\subsection{Simulation Model}

Our simulation setup consists of ONUs at the customers' premises, a centralized OLT, and an ODN that emulates a passive optical splitter/combiner, which splits the optical fiber cable running from the OLT to the ONUs. To check the impact on our algorithms from the number of ONUs in a PON system, we have created three different sets of scenarios, with 8 ONUs, 16 ONUs and 64 ONUs, respectively. The simulations involving 16 ONUs scenarios are further classified into two subcategories based on the distances from the ONUs to the OLT. One scenario is composed of 16 ONUs that are physically located at distances uniformly distributed between $18 \mathrm{~km}$ and $20 \mathrm{~km}$, while the other set of 16 ONUs are physically located at distances uniformly distributed between $2 \mathrm{~km}$ and $20 \mathrm{~km}$. In the downstream communication, the OLT broadcasts data to the ONUs, and each ONU filters the data sent to it and discards others. The upstream channel has a total capacity of 4 Gbps on four wavelengths, each one with a rate of 1 Gbps dynamically managed by the DBA. All ONUs are connected to their respective traffic sources and equipped with a packet generator over a link of $1 \mathrm{Gbps}$, thus avoiding possible bottlenecks. The maximum cycle time $\left(\delta_{\max }\right)$ is $1 \mathrm{~ms}$, and the sources generate self-similar traffic $[43,50]$ with Hurst parameter $\mathrm{H}=0.75$ and a mean packet rate that is adjusted according to varying offered load. The frame size follows a uniform distribution with a lower limit of 512 bits and an upper limit of 12,144 bits, thus realistically modeling Ethernet traffic [43].

Several scenarios are created for the simulations in order to evaluate the effect on the algorithms from LPT scheduling, WFQ-based differentiation, and laser tuning time. A guaranteed weight of a specified percentage of the system's total bandwidth capacity is allocated to some ONUs, thus causing them to have different QoS. A laser tuning time of LTT $=10 \mu \mathrm{s}$ is selected in reference to ITU-T G.989.2 specifications class 2 devices [27]. Traffic loads vary from $5 \%$ to $100 \%$ of the total load, where the maximum global offered load is $4 \mathrm{Gbps}$. The simulated algorithm sets are classified as IPACT, LPT, WFQ, and WFQLPT, depending on the configuration:

Set 1 . IPACT with four wavelengths at LTT $=0$ and $10 \mu \mathrm{s}$.

Set 2. $\quad$ LPT over IPACT with four wavelengths at LTT $=0$ and $10 \mu \mathrm{s}$.

Set 3. WFQ with four wavelengths at LTT $=0$ and $10 \mu \mathrm{s}$.

Set 4. LPT over WFQ (WFQLPT) with four wavelengths at LTT $=0$ and $10 \mu \mathrm{s}$

For the simulations performed using the IPACT and LPT algorithms, the ONUs have an equal share of the total bandwidth and transmit on four wavelengths. To evaluate the QoS, we introduced different weights into the WFQ and WFQLPT algorithms. The 16 ONUs are distributed in such a way that ONU 1 and ONU 2 have a guaranteed share of, $20 \%(800 \mathrm{Mbps}$ ) and 10\% (400 Mbps) respectively, and ONUs 3 to 16 each have 5\% (200 Mbps).

\subsection{Results}

In terms of throughput and queue delay, we evaluate the performance of our novel DBA algorithm in comparison with IPACT, which has been extended to support four wavelengths. The results and discussion of each parameter based on allocated weight are presented as follows.

\subsubsection{Throughput}

The throughput represents the average number of bits per unit time, measured in Mbps, and it includes the Ethernet header (destination and source addresses) and trailer (frame check sequence) that are successfully transmitted by the ONUs. In this subsection, we present the comparative performance of the four DBA algorithms in terms of throughput for the upstream link under varying offered loads.

Figure 2 shows the QoS differentiation of our algorithms by allocating different weights to the ONUs in order to see the effect of LPT scheduling on the algorithms. We 
separate the Max-Min-based algorithms (WFQ and WFQLPT) from IPACT and LPT because of uneven bandwidth allocated to different ONUs. Figure 2 (left) presents the results for IPACT and LPT transmitting on all four wavelengths at 0 LTT for all the ONUs. In this case, all ONUs have an equal share of the system, with each ONU having a share of 250 Mbps. Here, we see that both IPACT and LPT behave similarly, as they can transmit an equal amount of throughput up to $220 \mathrm{Mbps}$ before reaching saturation at an offered load of $210 \mathrm{Mbps}$. Thus, the introduction of LPT scheduling has no noticeable effect on IPACT in terms of throughput.
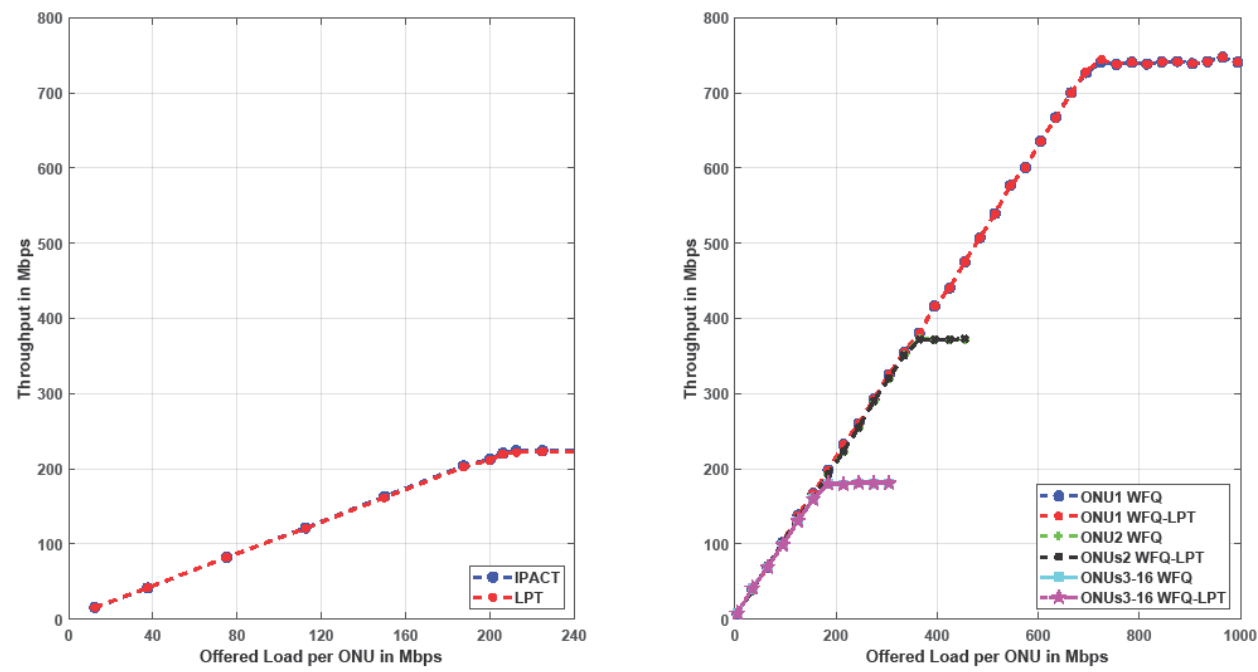

Figure 2. Throughput for all $16 \mathrm{ONUs}$ at LTT $=0 \mu \mathrm{s}$; IPACT $v s$. LPT (left) and WFQ $v s$. WFQLPT (right).

Figure 2 (right) shows the results for WFQ and WFQLPT with different weights. This scenario displays the QoS differentiation of the ONUs, with ONUs 1 and 2 having a share of $20 \%$ ( $800 \mathrm{Mbps}$ ) and $10 \%$ (400 Mbps), respectively; and ONUs 3 to 16 each have $5 \%$ (200 Mbps). The results show that ONU 1 can transmit up to $730 \mathrm{Mbps}$ at a bandwidth efficiency of $91 \%$ before reaching saturation at an offered load of $725 \mathrm{Mbps}$. ONU 2 can transmit up to $375 \mathrm{Mbps}$ at a bandwidth efficiency of $93.75 \%$ before reaching saturation at 360 Mbps. ONUs 3 to 16 can transmit up to $180 \mathrm{Mbps}$ at a bandwidth efficiency of $90 \%$ while reaching saturation at an offered load of $185 \mathrm{Mbps}$. Thus, the introduction of LPT has no noticeable effect on the WFQ algorithm in terms of throughput.

The scenarios in Figure 3 show the effect of LTT on the throughput for the four algorithms. Figure 3 (left) displays the results for ONU1, whose share of the resources (250 $\mathrm{Mbps}$ ) is equal to the remaining 15 ONUs under the IPACT and LPT algorithms at both $\mathrm{LTT}=0 \mu \mathrm{s}$ and LTT $=10 \mu \mathrm{s}$. With the ONUs transmitting on all four wavelengths, it can be seen that there is no noticeable difference in both IPACT and LPT for the two different LTTs (LTT $=0 \mu \mathrm{s}$ and LTT $=10 \mu \mathrm{s}$ ), as the ONUs are transmitting at a maximum throughput of $225 \mathrm{Mbps}$. Thus, the laser tuning time does not affect the throughput when ONUs have an equal allocation of $250 \mathrm{Mbps}$. 

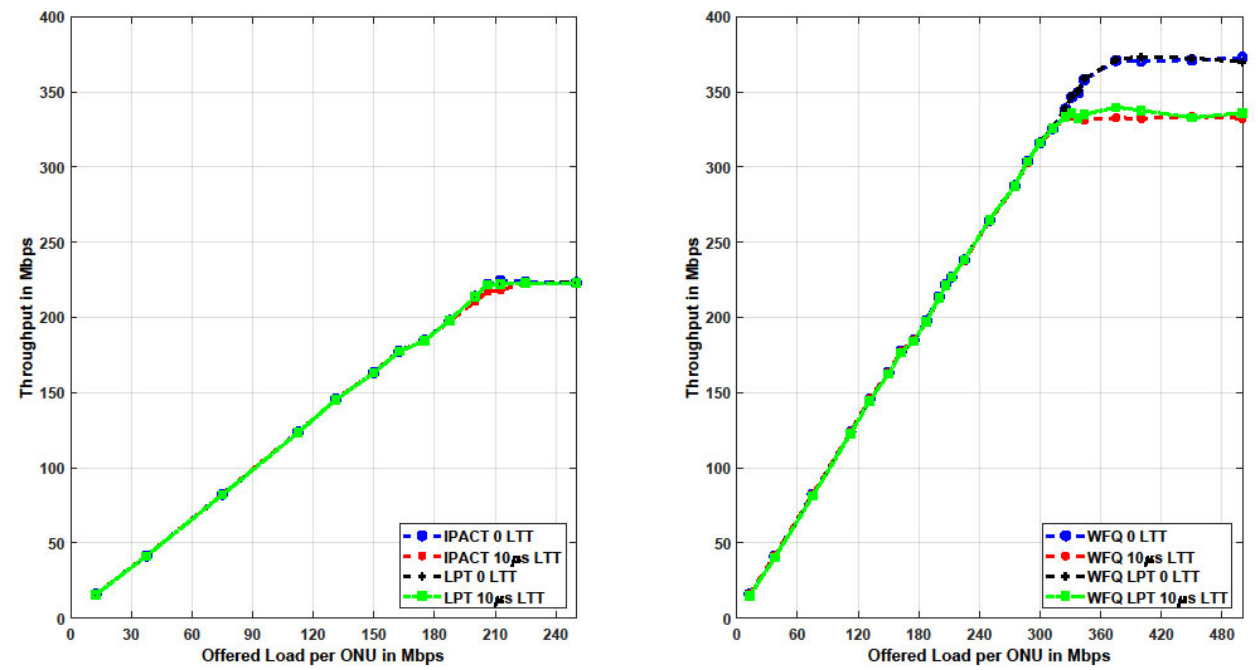

Figure 3. Throughput for ONU 1 at both LTT $=0 \mu$ s and LTT $=10 \mu$ s in a 16-ONU scenario; IPACT and LPT (left); and WFQ and WFQLPT (right).

In Figure 3 (right), we compare WFQ and WFQLPT at LTT $=0 \mu$ s and LTT $=10 \mu$ s for ONU1. In this case, ONU 1 has a share of $10 \%$ (400 Mbps) of the total resources, and the rest of the ONUs share the remaining $90 \%$. We can see that at higher offered load, there is a difference in the throughput between LTT $=0 \mu$ s and LTT $=10 \mu$ s for both WFQ and WFQLPT. While ONU 1 can transmit up to $370 \mathrm{Mbps}$ at LTT $=0$ for both WFQ and WFQLPT, it is only able to transmit up to $335 \mathrm{Mbps}$ when LTT of $10 \mu$ s is introduced before reaching saturation.

Figure 4 shows the results comparing the behavior of our system with different numbers of ONUs in the PON. We present the results for LPT and IPACT for 8 ONUs against 16 ONUs under LTT $=10 \mu \mathrm{s}$.
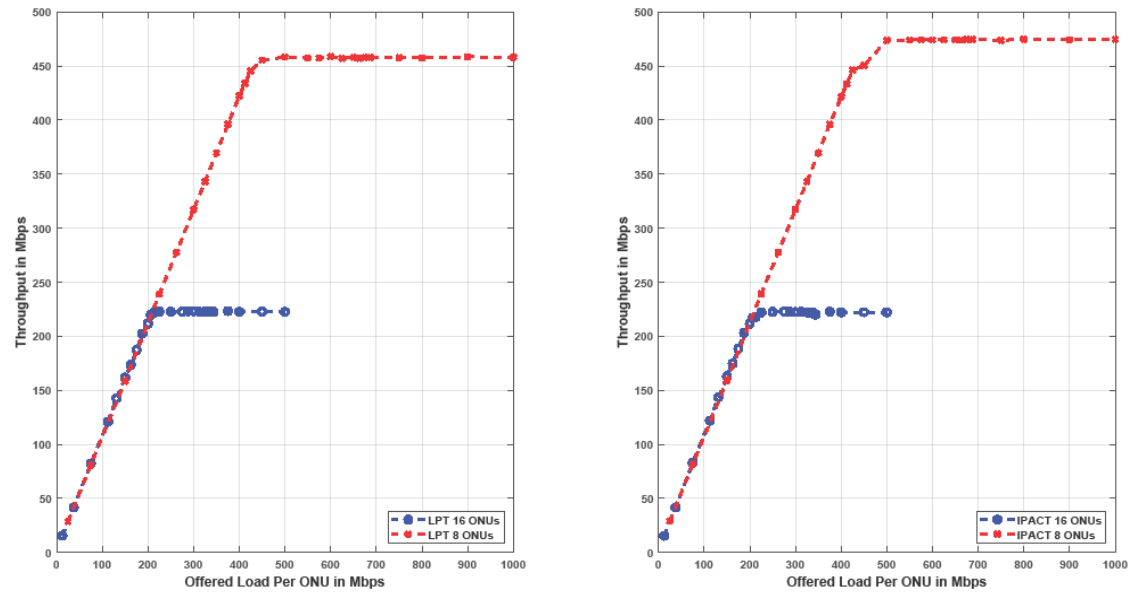

Figure 4. Throughput of all ONUs at LTT $=10 \mu$ s for 16 ONUs vs. 8 ONUs; LPT (left) and IPACT (right).

Figure 4 (left) shows that the throughput reaches $465 \mathrm{Mbps}$ in the 8-ONU system and $225 \mathrm{Mbps}$ in the 16-ONU system under the LPT algorithm. Figure 4 (right) shows that the throughput reaches $465 \mathrm{Mbps}$ with $8 \mathrm{ONUs}$ and $225 \mathrm{Mbps}$ with $16 \mathrm{ONUs}$ under the IPACT algorithm. These results show a proportional increase from $225 \mathrm{Mbps}$ to about $465 \mathrm{Mbps}$ 
when the ONUs decrease from 16 to 8 . These results show that LPT and IPACT behave similarly, regardless of the number of ONUs in the PON system.

In order to check the effect of the distance between the OLT and ONUs in our algorithms, we compare the results for the set of 16 ONUs that are scattered within a distance range of 2-20 km versus those within a distance range of $18-20 \mathrm{~km}$ from the ONUs to the OLT. Figure 5 (left) shows the throughput in the case of the LPT algorithm. As we can see, LPT behaves the same within both ranges, as it can transmit up to $220 \mathrm{Mbps}$ before reaching saturation at an offered load of $210 \mathrm{Mbps}$ at LTT $=10 \mu$ s.
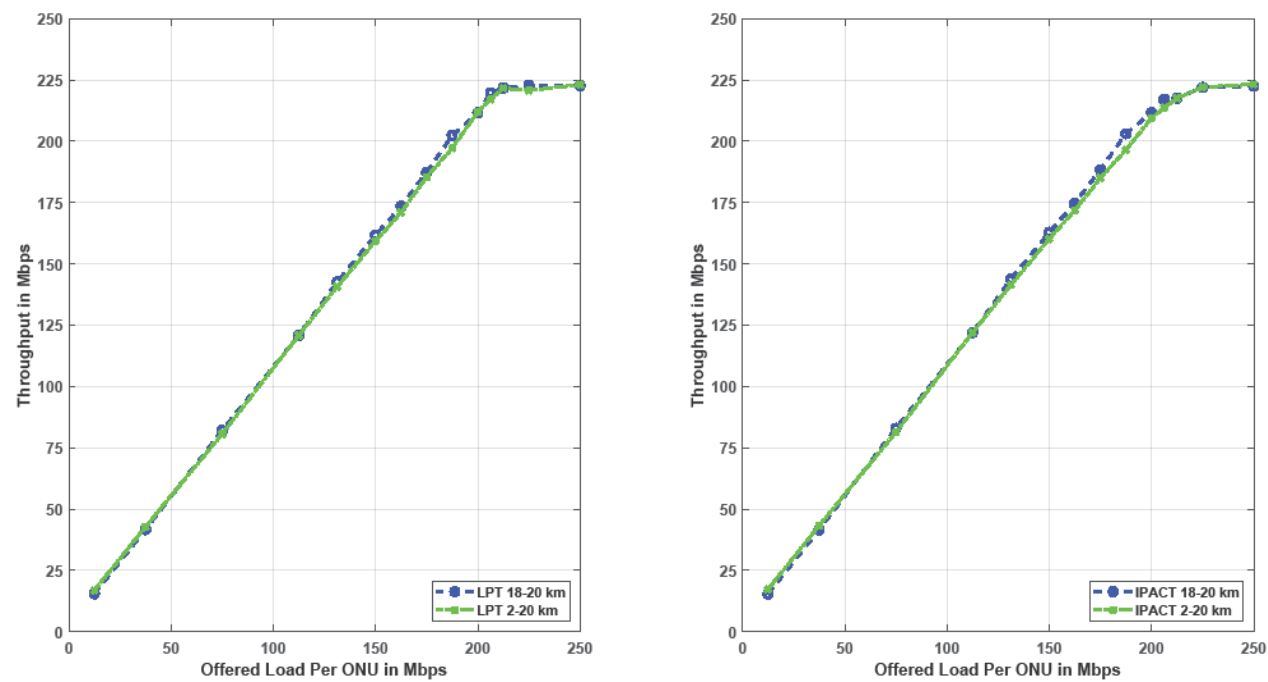

Figure 5. Throughput for all ONUs at LTT $=10 \mu$ s for a range of $18-20 \mathrm{~km} v s .2-20 \mathrm{~km}$ for LPT (left) and for IPACT (right).

Figure 5 (right) shows the results for ONUs within 18-20 km against 2-20 km from the OLT under the IPACT algorithm at LTT $=10 \mu \mathrm{s}$. As we can see, the IPACT algorithm has the same behavior within both distance ranges, as the ONUs can transmit up to 222 Mbps before reaching saturation at $225 \mathrm{Mbps}$.

Figure 6 shows the LPT's impact on the throughput in function of the distance and load for LTT $=0 \mu \mathrm{s}$. ONU 1 and ONU 4 are located at $2 \mathrm{~km}$ and $20 \mathrm{~km}$ from the OLT, respectively. Figure 6 (left) shows the CDF of the throughput for IPACT. Therefore, we can conclude that the range in which the ONUs are spread has no impact on the throughput of the system at low loads. For both IPACT and LPT at heavy loads, the ONUs closest to the OLT have the same behavior. In contrast, for the distant ONUs, LPT suffers a deviation of less than $10 \%$ in terms of IPACT. This is because LPT reduces the delay of the frames even if the system works at heavy loads. 

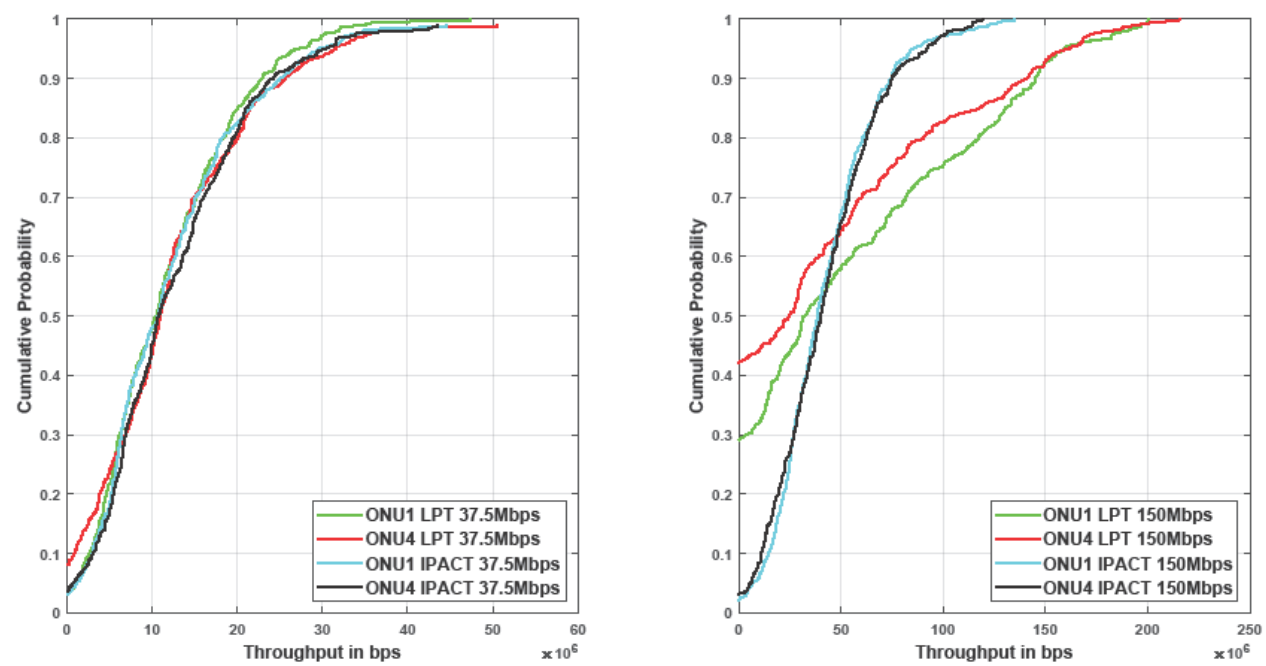

Figure 6. CDF of the throughput for ONU 1 and 4 at LTT $=0 \mu$ s; IPACT vs. LPT at low load (left) and heavy load (right).

\subsubsection{Queue Delay}

The queue delay measured in our simulations is the average packet waiting time in the ONU queues before being processed. The ONUs' queue delay is one of the components that forms the end-to-end delay, and the only one that is variable. In our scenario, packet transmission delay and propagation delay are negligible compared to the queue delay. We compare the queue delay for the IPACT, LPT, WFQ, and WFQLPT algorithms.

Figure 7 (left) shows the results for the average queue delay of all 16 ONUs for LPT versus IPACT at 0 LTT. In this scenario, all ONUs have an equal share of the system (250 $\mathrm{Mbps}$ ) and transmit on all four wavelengths. The results show that the ONUs have a much lower delay of $0.08 \mathrm{~ms}$ under the LPT versus the IPACT algorithm $(0.3 \mathrm{~ms})$. It can be seen that ONUs under LPT and IPACT reach saturation at the same offered load of around 200 Mbps.
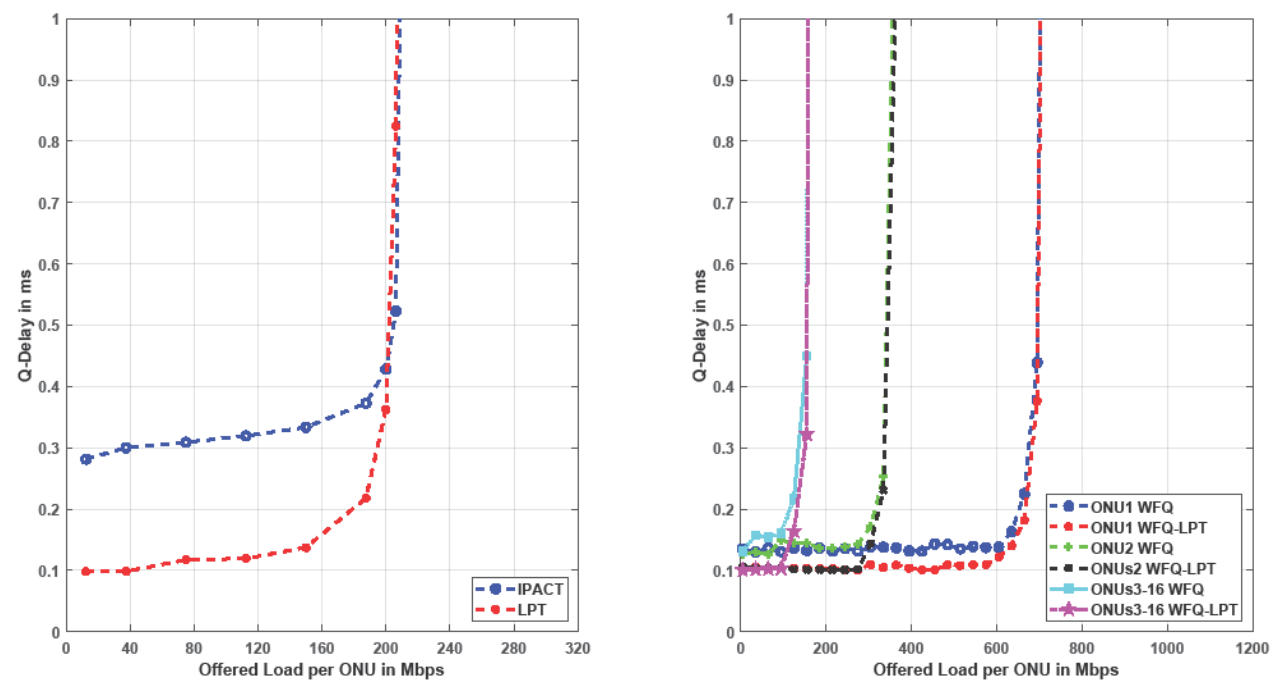

Figure 7. Queue delay for all ONUs at LTT =0 $\mu$ s; IPACT $v$ s. LPT (left) and WFQ vs. WFQLPT (right). 
Figure 7 (right) shows the results for ONUs under WFQ and WFQLPT using three different sets of weights (share of resources) for 0 LTT. ONU 1 has $800 \mathrm{Mbps}$, ONU 2 has about $400 \mathrm{Mbps}$, and ONUs 3 to 16 have $200 \mathrm{Mbps}$ each. We can see that the ONUs have lower delays under WFQLPT $(0.1 \mathrm{~ms})$ than WFQ $(0.15 \mathrm{~ms})$. In addition, the variation in the allocated resources for the ONUs causes them to have three different sets of saturation points with respect to the allocated resources.

Figure 8 presents the effect of LTT on queue delay for the four algorithms compared to the offered loads. Figure 8 (left) shows the results for ONU 1 with an allocation of 250 Mbps under IPACT, and under LPT at both LTT $=0 \mu$ s and LTT $=10 \mu$ s. We can see that IPACT at LTT $=0 \mu$ s has a slightly lower queue delay $(0.281 \mathrm{~ms})$ than when LTT $=10 \mu \mathrm{s}$ (0.296 ms).
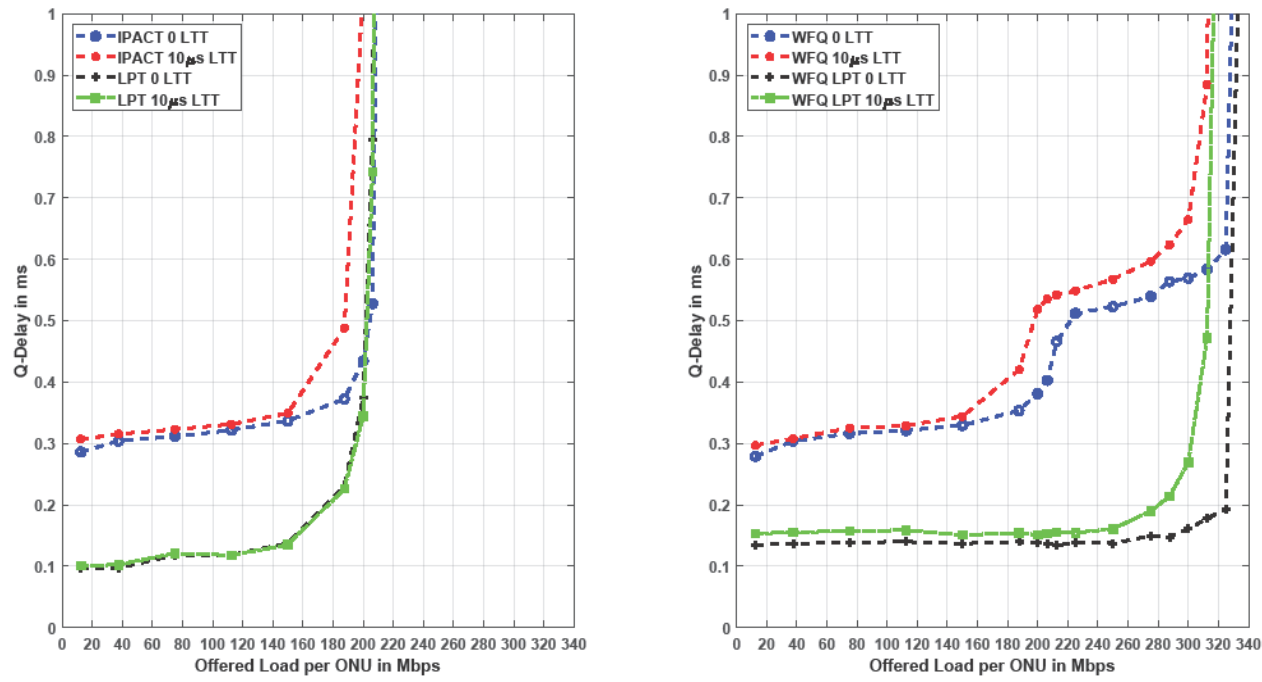

Figure 8. Queue delay for ONU 1 at both LTT $=0 \mu \mathrm{s}$ and LTT = $10 \mu \mathrm{s}$; IPACT and LPT (left); and WFQ and WFQLPT (right).

In Figure 8 (right), WFQ and WFQLPT are compared under LTT $=0 \mu$ s and LTT $=10$ $\mu$ s. In this case, ONU 1 has an allocation of 400 Mbps. For WFQLPT, the effect of LTT can be seen, as the queue delay is slightly lower when LTT $=0 \mu$ s in the working area $(0.279$ $\mathrm{ms}$ at an offered load of $15 \mathrm{Mbps})$ than when LTT $=10 \mu \mathrm{s}(0.297 \mathrm{~ms}$ at an offered load of $15 \mathrm{Mbps}$ ). If we compare the LPT queue delay with WFQLPT, we observe that the queue delay in the working area has increased by up to $50 \mu \mathrm{s}$. This increase is justified because the proposed WFQLPT algorithm offers quality of service, guarantees throughput, and thus minimizes delay. In the figure for WFQ with WFQLPT on the right, the former only guarantees throughput while the latter guarantees both parameters.

Figure 9 shows the results for the simulations of the IPACT and LPT algorithms, which were run to check the behavior of our system when different numbers of ONUs are connected. We compare the queue delay for the three scenarios with 64 ONUs vs. 16 ONUs vs. 8 ONUs at LTT $=10 \mu \mathrm{s}$. The results show that, apart from the expected rescaling of the saturation point, the number of ONUs does not have any important impact, and the LPT and IPACT algorithms behave similarly. Figure 9 (left) shows that the queue delay under the LPT algorithm is kept at almost the same value between $0.1 \mathrm{~ms}$ and $0.15 \mathrm{~ms}$ in the three scenarios until it reaches saturation at an offered load of approximately $50 \mathrm{Mbps}$, in the case of $64 \mathrm{ONUs}, 200 \mathrm{Mbps}$, in the case of $16 \mathrm{ONUs}$, and of $400 \mathrm{Mbps}$ for the 8 ONUs. 

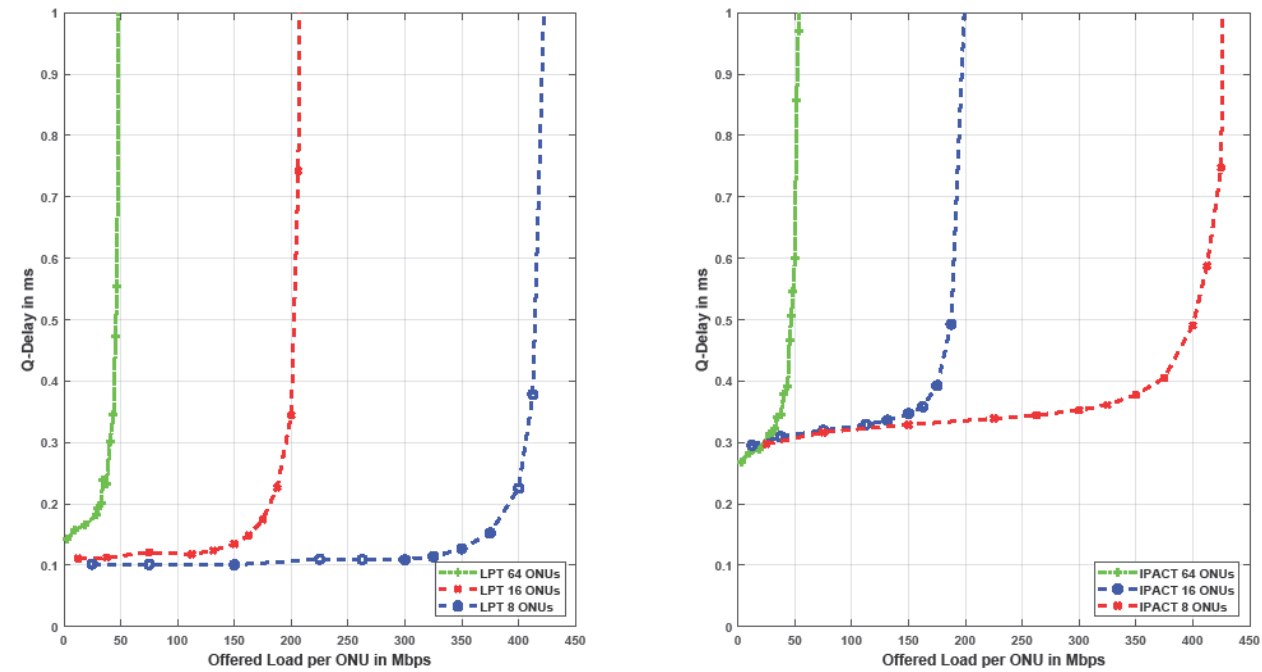

Figure 9. Average queue delay for all ONUs at LTT = $10 \mu$ s for 64 ONUs, 16 ONUs and 8 ONUs; LPT (left) and IPACT (right).

Figure 9 (right) shows that, under the IPACT algorithm, the queue delay is similar for the three scenarios, as it is kept at around $0.3 \mathrm{~ms}$ until reaching an offered load of 48 Mbps in the case of 64 ONUs, $190 \mathrm{Mbps}$, in the case of 16 ONUs, and of $400 \mathrm{Mbps}$ with 8 ONUs. These results confirm that the three sets of ONUs $(64,16$, and 8$)$ have similar behaviors under the IPACT and LPT algorithms.

In Figure 10, we evaluate the performance of the LPT and IPACT algorithms in two scenarios with 16 ONUs: in the first, the ONUs are scattered within a distance range of 2$20 \mathrm{~km}$ to the OLT; and in the second, the distance range is $18-20 \mathrm{~km}$. Figure 10 (left) shows the queue delay results for the LPT algorithm, and it is evident that the behavior is the same in both ranges, as the queue delay is kept at $0.11 \mathrm{~ms}$ before reaching saturation at 190 Mbps.
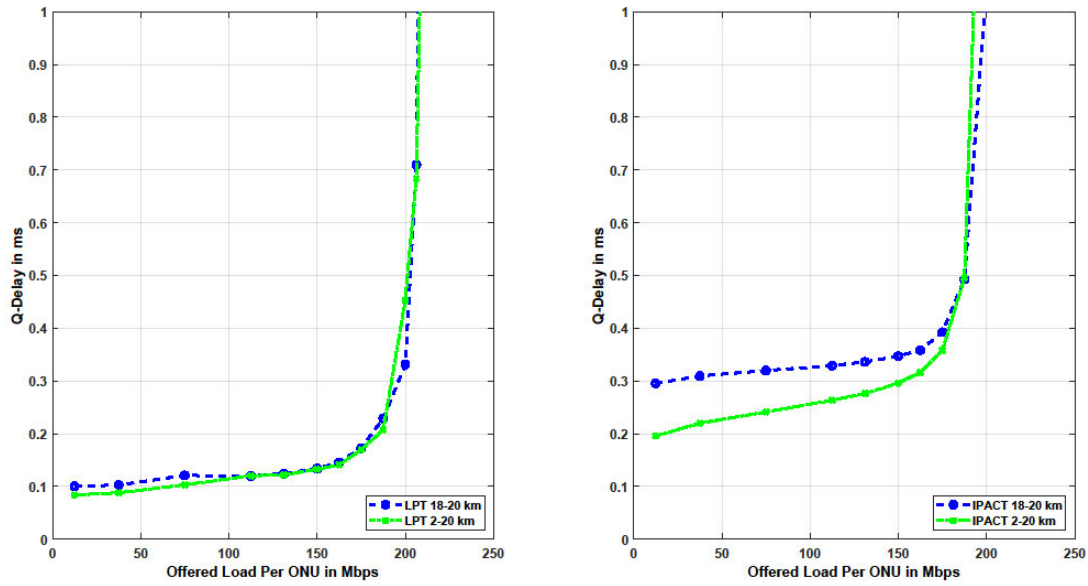

Figure 10. Queue delay for a 16-ONU system with a distance range of 2-20 km vs. $18-20 \mathrm{~km}$ at LTT = $10 \mu \mathrm{s}$; LPT (left) and IPACT (right).

Figure 10 (right) shows the IPACT algorithm's queue delay results for the set of ONUs within 2-20 km vs. 18-20 km. It can be deduced that the queue delay in the 18-20 $\mathrm{km}$ range goes from $0.3 \mathrm{~ms}$ until reaching $0.35 \mathrm{~ms}$ near the saturation point at offered loads 
of $190 \mathrm{Mbps}$. Meanwhile, in the 2-20 km range scenario, the queue delay goes from $0.2 \mathrm{~ms}$ to $0.3 \mathrm{~ms}$ near the saturation point at offered loads of $190 \mathrm{Mbps}$.

Figure 11 shows the Cumulative Distribution Function (CDF) of the queue delay for ONU 1 and ONU 4 at offered loads of $37.5 \mathrm{Mbps}$ and $150 \mathrm{Mbps}$ under the IPACT and LPT algorithms for LTT $=10 \mu \mathrm{s}$. ONU 1 and ONU4 are located at, respectively, $2.61 \mathrm{~km}$ and $17.43 \mathrm{~km}$ from the OLT in the 16-ONU scenario. Figure 11 (left) shows that, at low load, LPT's margin of improvement is between $50 \mu$ s to $100 \mu$ s for IPACT, while under LPT the impact of the distance is limited to below $25 \mu \mathrm{s}$.
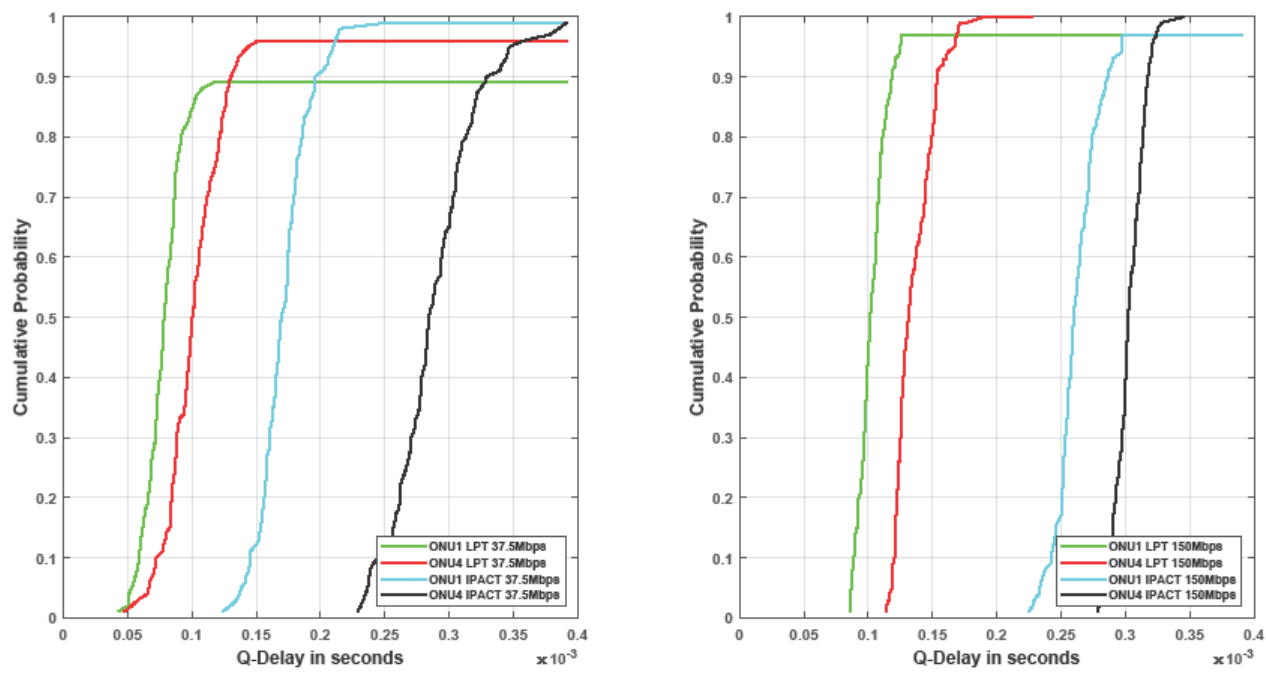

Figure 11. CDF of the queue delay for ONU 1 and ONU 4 under the IPACT and LPT algorithms for LTT $=10 \mu$ s affered loads of $37.5 \mathrm{Mbps}$ (left) and $150 \mathrm{Mbps}$ (right).

In Figure 11 (right), we show the queue delay for both ONU 1 and ONU 4 at an offered load of $150 \mathrm{Mbps}$. For heavy loads, the improvement of LPT with respect to IPACT is more evident, as it increases the difference by $150 \mu \mathrm{s}$. Furthermore, the variability of the queue delay for heavy loads and different distances is kept at around $40 \mu \mathrm{s}$. This delay is similar to that of low loads. Thus, it can be deduced from Figures 10 and 11 that even though the queue delay increases together with the distance between the ONU and OLT, the difference in the queue delay narrows as the offered load increases.

\subsection{Discussion of the Results}

The results show four main aspects. First, we have our analysis on the influence of laser tuning time on IPACT, in terms of throughput, and queue delay as a function of the system load and the distance between the ONUs and the OLT. Second, the LPT optimizes the queue delay more than IPACT in all the above scenarios. Third, as the WFQ guarantees a throughput for each user, we have evaluated the impact when the LTT is introduced. Finally, the performance of the WFQLPT has been evaluated, which guarantees a minimum queue delay and the bandwidth requested by the different ONUs.

We can achieve an average bandwidth efficiency of about $85 \%$ in the upstream link, which conforms to the minimum absolute efficiency that is stipulated in [51]. The inefficiency in the system is a result of overhead from encapsulation, such as control message overhead (which represents bandwidth lost to GATE and REPORT message exchanges between the ONUs and OLT), guard band overhead, discovery overhead, and frame delineation overhead [51]. The overhead consumes up to $16.37 \%$ of the bandwidth, with the minimum throughput being $836.3 \mathrm{Mbps}$ on a 1 Gbps link. 
Introducing a realistic LTT of $10 \mu$ s provokes a noticeable decrease in the throughput and an increase in the queue delay. The effect on the throughput is seen only at higher loads, above $320 \mathrm{Mbps}$ ( $80 \%$ offered), with the delay introduced as a result of LTT reducing the throughput capability of the ONUs by $10 \%$ compared to when there is no LTT, thus reducing the bandwidth utilization of the system. The effect of LTT is not noticed at lower loads because the system is not operating at nearly full capacity. As such, the system can transmit up to the maximum allowable throughput. The effect of LTT on the queue delay is seen only at the point where the queue delay starts skyrocketing, which is at a much higher offered load when the LTT is 0 than when the LTT is $10 \mu \mathrm{s}$. At lower loads, the queue delay is kept minimal and comparable to when there is no LTT applied.

The number of ONUs connected to the OLT does not in any way affect the performance of the DBA algorithms in terms of queue delay and throughput. When the resources are to be shared equally among the ONUs in the PON, the algorithms behave the same way, with each ONU's throughput and queue delay having similar values. The distance between the ONU and the OLT within the maximum allowed distance $(20 \mathrm{~km})$ of PON does not have any impact on the throughput. It does not matter where the ONU is located in the PON, the throughput will still be the same. However, the queue delay is affected and it decreases as the ONUs become closer to the OLT. However, the impact of the distance between the ONU and the OLT decreases as the offered load increases.

These results emphasize the need to consider LTT when designing DBA and evaluating its performance in order to obtain realistic results and model the behavior of a system whose delay requirements are within a band of $1 \mathrm{~ms}$ to $100 \mathrm{~ms}$ [52], which is what critical services demand in $5 \mathrm{G}$ networks.

The LPT scheme is introduced to solve the problem of minimizing the total finish time when scheduling requests on multiple wavelengths. When LPT is applied to the IPACT algorithm, the queue delay is reduced (by 73\%), but there is no noticeable effect on the throughput. Applying LPT to the WFQ algorithm gives us the WFQLPT algorithm, which is a hybrid that combines the low delay of LPT with the QoS differentiation provided by WFQ. Therefore, WFQLPT achieves QoS differentiation and proves to be superior to WFQ in terms of delay, which is reduced by approximately $33 \%$. In terms of throughput, there is no noticeable difference between WFQLPT and WFQ.

When implementing QoS differentiation in WFQ and WFQLPT algorithms, the ONUs with higher priority obtain their share and the remaining ONUs obtain a fair share of the resources without any of them being starved. The introduction of QoS based on WFQ reduces wasted bandwidth because the bandwidth utilization is in the region of $91 \%$, which is higher than when no QoS is applied ( $88 \%)$, and there is no noticeable impact on the delay.

\section{Conclusions}

TWDM-based PON is a promising technology with great potential for providing the high bandwidth capacity and low latency required by emerging services. TWDM-PON DBA algorithms need to take into account the laser tuning time (LTT), which is often ignored. In this paper, we have presented a WFQLPT, QoS-aware algorithm that considers LTT. Our algorithm builds on IPACT by adding the capability of supporting four wavelengths. We apply a scheduling mechanism based on the LPT scheme that arranges the requests from the ONUs in descending order before being scheduled on the assigned wavelengths in accordance with the NASC principle, thus reducing delay. As IPACT is known to lack QoS capability, we have introduced weight-based QoS differentiation based on Max-Min Weighted Fair Share in order to ensure fair sharing of resources. We evaluated our approach through simulations, and our results show that the bandwidth is shared fairly among the users while wavelengths are allocated in a more balanced manner. Introducing WFQ guarantees the allocation of resources based on the Service Level Agreement (SLA) while keeping delay bounded. We can see the effect of LPT in reducing the average packet delay on IPACT by $73 \%$ and on the WFQ algorithm by $33 \%$. We have 
also shown that the delay introduced as a result of LTT gives the system more realistic behavior in terms of the throughput and queue delay.

This paper opens up a new horizon of research on the implementation of the DBA algorithm while focusing on efficient energy utilization in order to save power, which is a worthwhile contribution, given the predominant role of PONs in next-generation networks. We plan to introduce power-saving features such as laser doze/sleep mode [53] and further exploit the laser tuning time to achieve optimal results while keeping the delay minimal. We also intend to enhance the algorithm by implementing it in a just-in-time manner in order to further reduce the delay [33]. Furthermore, we will work on improving the management architecture of TWDM-PON by introducing the software-defined networking principle to decouple the OLT and move the DBA functions to a centralized controller, which will thus manage the network with flexibility [54]. An interesting direction for future research will be to consider Long-Reach Passive Optical Networks (LRPON), with a multi-thread polling scheme to enhance their operations.

Author Contributions: Conceptualization, A.H. and M.Z.; methodology, A.H. and M.Z.; software, A.H., M.Z. and A.B.; validation, D.R., S.S., and J.R.P.; formal analysis, A.H., and M.Z.; investigation, A.H. and M.Z.; resources, A.H. and M.Z.; writing-original draft preparation, A.H. and M.Z.; writingreview and editing, A.H., M.Z., D.R., S.S. and J.R.P.; visualization, A.H., M.Z., D.R., S.S. and J.R.P.; supervision, D.R., S.S., J.R.P., and A.B. All authors have read and agreed to the published version of the manuscript.

Funding: This work has been supported by the Agencia Estatal de Investigación of Spain under project PID2019-108713RB-C51/AEI/10.13039/501100011033.

Data Availability Statement: Not applicable.

Conflicts of Interest: The authors declare no conflict of interest.

\section{References}

1. Taylor, R.; Baron, D.; Schmidt, D. The world in 2025-Predictions for the next ten years. In Proceedings of the 10th International Microsystems, Packaging, Assembly and Circuits Technology Conference (IMPACT), Taipei, Taiwan, 21-23 October 2015; pp. 192-195.

2. Hadi, M.; Bhar, C.; Agrell, E. General QoS-aware scheduling procedure for passive optical networks. J. Opt. Commun. Netw. 2020, 12, 217-226.

3. Pizzinat, A.; Chanclou, P.; Saliou, F.; Diallo, T. Things You Should Know About Fronthaul. J. Light. Technol. 2015, 33, 1077-1083, doi:10.1109/jlt.2014.2382872.

4. Nakayama, Y.; Hisano, D. Wavelength and Bandwidth Allocation for Mobile Fronthaul in TWDM-PON. IEEE Trans. Commun. 2019, 67, 7642-7655, doi:10.1109/tcomm.2019.2939319.

5. Kondepu, K.; Valcarenghi, L.; Castoldi, P. Balancing the impact of ONU tuning overhead in reconfigurable TWDM-PONs: An FPGA-based evaluation. In Proceedings of the IEEE Global Communications Conference (GLOBECOM), San Diego, CA, USA, 6-10 December, 2015; pp. 1-6.

6. Memon, K.A.; Mohammadani, K.H.; Shaikh, A.; Ullah, S.; Zhang, Q.; Das, B.; Ullah, R.; Tian, F.; Xin, X. Demand forecasting DBA algorithm for reducing packet delay with efficient bandwidth allocation in XG-PO. Electronics 2019, 8, 147.

7. Ruffini, M.; Achouche, M.; Arbelaez, A.; Bonk, R.; Di Giglio, A.; Doran, N.J.; Furdek, M.; Jensen, R.; Montalvo, J.; Parsons, N.; et al. Access and Metro Network Convergence for Flexible End-to-End Network Design. J. Opt. Commun. Netw. 2017, 9, 524-535, doi:10.1364/JOCN.9.000524.

8. Rafiq, A.; Hayat, M.F. QoS-Based DWBA Algorithm for NG-EPON. Electronics 2019, 8, 230.

9. Liu, X.; Deng, N.; Zhou, M.; Wang, Y.; Tao, M.; Zhou, L.; Li, S.; Zeng, H.; Megeed, S.; Shen, A.; et al. Enabling Technologies for 5G-Oriented Optical Networks. In Proceedings of the Optical Fiber Communications Conference and Exhibition (OFC), San Diego, CA, USA, 3-7 March, 2019; pp. 1-3.

10. Afraz, N.; Slyne, F.; Gill, H.; Ruffini, M. Evolution of Access Network Sharing and Its Role in 5G Networks. Appl. Sci. 2019, 9, 4566, doi:10.3390/app9214566.

11. Nakayama, Y.; Uzawa, H.; Hisano, D.; Ujikawa, H.; Nakamura, H.; Terada, J.; Otaka, A. Efficient DWBA Algorithm for TWDMPON with Mobile Fronthaul in 5G Networks. In Proceedings of the IEEE Global Communications Conference (GLOBECOM), Singapore, 4-8 December 2017; pp. 1-6.

12. Dixit, A.; Lannoo, B.; Colle, D.; Pickavet, M.; Demeester, P. Dynamic bandwidth allocation with optimal wavelength switching in TWDM-PONs. In Proceedings of the 15th International Conference on Transparent Optical Networks (ICTON), Cartagena, Spain, 23-27 June 2013; pp. 1-4. 
13. Park, C.; Min, J.; Kim, S. Semi-passive optical front-haul supporting channel monitoring and link protection for the cloud radio access network. IET Commun. 2019, 13, 442-448, doi:10.1049/iet-com.2018.5726.

14. Xue, L.; Yi, L.; Ji, H.; Li, P.; Hu, W. Symmetric 100-Gb/s TWDM-PON in O-Band Based on 10G-Class Optical Devices Enabled by Dispersion-Supported Equalization. J. Light. Technol. 2017, 36, 580-586, doi:10.1109/jlt.2017.2777498.

15. Jaffer, S.S.; Hussain, A.; Qureshi, M.A.; Khawaja, W.S. Towards the Shifting of 5G Front Haul Traffic on Passive Optical Network. Wirel. Pers. Commun. 2020, 112, 1549-1568, doi:10.1007/s11277-020-07115-6.

16. Liem, A.T.; Hwang, I.-S.; Nikoukar, A.; Pakpahan, A.F. SD-Enabled Mobile Fronthaul Dynamic Bandwidth and Wave-length Allocation (DBWA) Mechanism in Converged TWDM-EPON Architecture. In Proceedings of the 6th International Conference on Cyber and IT Service Management (CITSM), Parapat. Indonesia, 7-9 August 2018; pp. 1-6.

17. Zhang, J.; Ansari, N. Scheduling Hybrid WDM/TDM Passive Optical Networks with Nonzero Laser Tuning Time. IEEE/ACM Trans. Netw. 2010, 19, 1014-1027, doi:10.1109/TNET.2010.2093150.

18. Buttaboni, A.; de Andrade, M.; Tornatore, M.; Pattavina, A. Dynamic bandwidth and wavelength allocation with coexist-ing transceiver technology in WDM/TDM PONs. Opt. Switch. Netw. 2016, 21, 31-42.

19. Ojeyinka, T.O. Bin packing algorithms with applications to passenger bus loading and multiprocessor scheduling problems. Commun. Appl. Electron. 2015, 2, 38-44.

20. Marbach, P. Priority service and max-min fairness. In Proceedings of the Twenty-First Annual Joint Conference of the IEEE Computer and Communications Societies, New York, NY, USA, 23-27 June 2002; Volume 1, pp. 266-275.

21. Kramer, G.; Mukherjee, B.; Pesavento, G. Interleaved Polling with Adaptive Cycle Time (IPACT): A Dynamic Bandwidth Distribution Scheme in an Optical Access Network. Photon. Netw. Commun. 2002, 4, 89-107, doi:10.1023/a:1012959023043.

22. Horvath, T.; Munster, P.; Vojtech, J. Deployment of PON in Europe and Deep Data Analysis of GPON. Telecommun. Syst. Princ. Appl. Wirel. Opt. Technol. 2019, 56-76, doi:10.5772/intechopen.82679.

23. Law, D.; Dove, D.; D'Ambrosia, J.; Hajduczenia, M.; Laubach, M.; Carlson, S. Evolution of ethernet standards in the IEEE 802.3 working group. IEEE Commun. Mag. 2013, 51, 88-96, doi:10.1109/MCOM.2013.6576344.

24. IEEE Standards Association, IEEE Standard for Ethernet, IEEE Std 802.3-2018 (Revision of IEEE Std 802.3-2015), IEEE, 31 August 2018. Available online: https://ieeexplore.ieee.org/document/8457469 (accessed on 29 April 2021).

25. ITU-T Recommendation, G.9807.1, 10-Gigabit-Capable Symmetric Passive Optical Network (XGS-PON), International Telecommunications Union, 1 June 2016. Available online: https://www.itu.int/rec/T-REC-G.9807.1/en (accessed on 29 April 2021).

26. IEEE Standards Association, 802.3ca-2020 Approved Draft Standard for Ethernet Amendment, IEEE, June 2020. Available online: https://standards.ieee.org/standard/802_3ca-2020.html (accessed on 24 June 2020).

27. ITU-T Recommendation G.989.2, 40-Gigabit-Capable Passive Optical Networks 2 (NG-PON2): Physical Media Dependent (PMD) Layer Specification, International Telecommunications Union, February 2019. Available online: https://www.itu.int/rec/T-REC-G.989.2 (accessed on 29 April 2021).

28. Wey, J.S.; Nesset, D.; Valvo, M.; Grobe, K.; Roberts, H.; Luo, Y.; Smith, J. Physical layer aspects of NG-PON2 standards-Part 1: Optical link design. IEEE/OSA J. Opt. Commun. Netw. 2016, 8, 33-42.

29. ITU-T Recommendation G.984.6, Gigabit-Capable Passive Optical Networks (GPON): Reach Extension, International Telecommunications Union, March 2008. Available online: https://www.itu.int/rec/T-REC-G.984.6-200803-I/en (accessed on 29 April 2021).

30. ITU-T Recommendation G.9807.2, 10 Gigabit-Capable Passive Optical Networks (XG(S)-PON): Reach Extension, International Telecommunications Union, August 2017. Available online: https://www.itu.int/rec/T-REC-G.9807.2/en (accessed on 29 April 2021).

31. ITU-T Work Programme, Work Item G.9807.3 (ex G.SuperPON). Available online: https://www.itu.int/ITU-T/workprog/wp_item.aspx?isnD15208 (accessed on 29 April 2021).

32. DeSanti, C.; Du, L.; Guarin, J.; Bone, J.; Lam, C. Super-PON: An evolution of access networks. Opt. Commun. Netw. 2020, 12, D66-D76.

33. McGarry, M.P.; Reisslein, M.; Colbourn, C.J.; Maier, M.; Aurzada, F.; Scheutzow, M. Just-in-time scheduling for multichan-nel EPON. Lightwave Technol. 2008, 26, 1204-1216.

34. Dixit, A.; Lannoo, B.; Colle, D.; Pickavet, M.; Demeester, P.; Abhishek, D. Energy efficient DBA algorithms for TWDM-PONs. In Proceedings of the 17th International Conference on Transparent Optical Networks (ICTON), Budapest, Hungary, 5-9 July 2015; pp. 1-5.

35. Kanonakis, K.; Tomkos, I. Improving the efficiency of online upstream scheduling and wavelength assignment in hybrid WDM/TDMA EPON networks. IEEE J. Sel. Areas Commun. 2010, 28, 838-848, doi:10.1109/JSAC.2010.100809.

36. Dixit, A.; Colle, D.; Lannoo, B.; Demeester, P.; Pickavet, M. Novel DBA algorithm for energy efficiency in TWDM-PONs. In Proceedings of 9th European Conference and Exhibition on Optical Communication (ECOC), London, UK, 22-26 September 2013; pp. 1-3.

37. Wang, W.; Guo, W.; Hu, W. Adaptive wavelength allocation pattern for an online DWBA in the NG-EPON. OSA Contin. 2018, 1, 690-702, doi:10.1364/osac.1.000690.

38. Zaouga, A.; De Sousa, A.; Najja, M.; Monteiro, P. Low Latency Dynamic Bandwidth Allocation Algorithms for NG-PON2 to Support 5G Fronthaul and Data Services. In Proceedings of the 21st International Conference on Transparent Optical Networks (ICTON), Angers, France, 9-13 July 2019; pp. 1-4. 
39. Wang, H.; Su, S.; Gu, R.; Ji, Y. A minimum wavelength tuning scheme for dynamic wavelength assignment in TWDM-PON. In Proceedings of the 7th IEEE International Conference on Software Engineering and Service Science (ICSESS), Beijing, China, 26-28 August 2016; pp. 1-3.

40. Zhang, H.; Zhang, M.; Liu, X.; Wang, D.; Jiang, L. A Multi-OLTs and Virtual Passive Optical Network for Hybrid Net-work. In Proceedings of the 7th IEEE International Conference on Software Engineering and Service Science (ICSESS), Beijing, China, 26-28 August 2016; pp. 1009-1012.

41. Zhang, L.; Qi, J.; Wei, K.; Zhang, W.; Feng, Y.; Hou, W. High-priority first dynamic wavelength and bandwidth allocation algorithm in TWDM-PON. Opt. Fiber Technol. 2019, 48, 165-172, doi:10.1016/j.yofte.2018.12.029.

42. Gravalos, I.; Yiannopoulos, K.; Papadimitriou, G.; Varvarigos, E.A. A modified max-min fair dynamic bandwidth alloca-tion algorithm for XG-PONs. In Proceedings of the 19th European Conference on Networks and Optical Communications (NOC), Milan, Italy, 4-6 June 2014; pp. 57-62.

43. Navarro, M.C. Study and Implementation of New DBA for WDM-PON. Master's Thesis, Universitat Politècnica de Cata-lunya (UPC), Barcelona, Spain, 2010.

44. Agrawal, T.K.; Sahu, A.; Ghose, M.; Sharma, R. Scheduling chained multiprocessor tasks onto large multiprocessor system. Computing 2017, 99, 1007-1028, doi:10.1007/s00607-017-0543-z.

45. Grigoriu, L. Approximation for Scheduling on Parallel Machines with Fixed Jobs or Unavailability Periods. Sched. Probl. New Appl. Trends 2020, 1-17, doi:10.5772/intechopen.89694.

46. Lee, C.-Y.; Massey, J.D. Multiprocessor scheduling: Combining LPT and MULTIFIT. Discret. Appl. Math. 1988, 20, 233-242, doi:10.1016/0166-218x(88)90079-0.

47. Pinedo, M.; Hadavi, K. Scheduling: Theory, Algorithms and Systems Development. In Operations Research Proceedings 1991; Springer: Berlin/Heidelberg, Germany, 1992; pp. 35-42.

48. Li, G.; Qian, Y.; Yang, Y.R. On max-min fair allocation for multi-source transmission. ACM Sigcomm Comput. Commun. Rev. 2019, 48, 2-8, doi:10.1145/3310165.3310167.

49. Alkallak, I.N. A Modified for Largest Processing Time Scheduling Algorithm in Multiprocessor. Tikrit J. Pure Sci. 2011, 16, 280283.

50. Melo, E.F.; de Oliveira, H.M. An Overview of Self-Similar Traffic: Its Implications in the Network Design. Rev. Tecnol. Inf. E Comun. 2020, 9, 38-46.

51. Kramer, G. How efficient is EPON? White Paper. Available online: https://www.ieee802.org/3/efm/public/p2mp_email/pdf00001.pdf (accessed on 29 April 2021).

52. Parvez, I.; Rahmati, A.; Guvenc, I.; Sarwat, A.I.; Dai, H. A Survey on Low Latency Towards 5G: RAN, Core Network and Caching Solutions. IEEE Commun. Surv. Tutor. 2018, 20, 3098-3130, doi:10.1109/comst.2018.2841349.

53. Khalili, H.; Rincón, D.; Sallent, S.; Piney, J.R. An Energy-Efficient Distributed Dynamic Bandwidth Allocation Algorithm for Passive Optical Access Networks. Sustainability 2020, 12, 2264.

54. Zehri, M.; Haastrup, A.; Rincón, D.; Piney, J.R.; Sallent, S.; Bazzi, A. Leveraging SDN-Based Management for Improved Traffic Scheduling in PONs. In Proceedings of the 22nd International Conference on Transparent Optical Networks (ICTON), Bari, Italy, 19-23 July 2020; pp. 1-4. 